\title{
Abstract \\ The Cosmological Model Based on the Uncertainty-Mediated Dark Energy
}

\section{Yurii Dumin}

Citation: Dumin, Y. The

Cosmological Model Based on the Uncertainty-Mediated Dark Energy. Phys. Sci. Forum 2021, 2, 37. https:// doi.org/10.3390/ECU2021-09515

Academic Editor: Yifu Cai

Published: 19 March 2021

Publisher's Note: MDPI stays neutral with regard to jurisdictional claims in published maps and institutional affiliations.

Copyright: (C) 2021 by the author. Licensee MDPI, Basel, Switzerland. This article is an open access article distributed under the terms and conditions of the Creative Commons Attribution (CC BY) license (https:/ / creativecommons.org/licenses/by/ $4.0 /)$.

\author{
Sternberg Astronomical Institute of Lomonosov Moscow State University, 119991 Moscow, Russia; \\ dumin@yahoo.com \\ + Presented at the 1st Electronic Conference on Universe, 22-28 February 2021; Available online: \\ https://ecu2021.sciforum.net/.
}

\begin{abstract}
The existence of the effective Lambda-term is a commonly accepted paradigm of modern cosmology, but the physical essence of this quantity remains absolutely unknown, and its numerical values are drastically different in the early and modern universe. In fact, the Lambda-term is usually introduced in the literature either by postulating arbitrary additional terms in the Lagrangians or by employing the empirical equations of state. In our recent series of papers (Yu.V. Dumin. Grav. and Cosmol., v.25, p.169 (2019); v.26, p. 259 (2020); v.27, in press (2021)), we tried to provide a more rigorous physical basis for the effective Lambda-term, starting from the time-energy uncertainty relation in the Mandelstam-Tamm form, which is appropriate for the long-term evolution of quantum systems. This results in the time-dependent Lambda-term, decaying as $1 / \mathrm{t}$. The uncertainty-mediated cosmological model possesses a number of specific features, some of which look rather appealing: (1) While the standard cosmology involves a few very different stages (governed by the Lambda-term, radiation, dustlike matter, and again the Lambda-term), our model provides a universal description of the entire evolution of the universe by the same "quasi-exponential" function. (2) As follows from the analysis of causal structure, the present-day cosmological horizon comprises a single domain developing from the Bing Bang. Therefore, the problems of the homogeneity and isotropy of matter, the absence of topological defects, etc. should be naturally resolved. (3) Besides, our model naturally explains the observed approximately flat 3D space, i.e., the solution with zero curvature is formed "dynamically", starting from the arbitrary initial conditions. (4) The age of the universe turns out to be much greater than in the standard cosmology; but this should not be a crucial drawback, because the most of problems are associated with insufficient rather than excessive age of the universe.
\end{abstract}

Keywords: cosmology; Dark Energy; quantum uncertainty relation

Supplementary Materials: The supplementary file is available at https:/ / www.mdpi.com/article/ 10.3390/ECU2021-09515/s1.

Data Availability Statement: The data are available at: https://link.springer.com/article/10.1134\% 2FS0202289319020051, https:/ /link.springer.com/article/10.1134\%2FS0202289320030068. 